Adhesive contact between a graphene sheet and a nano-scale corrugated surface

This article has been downloaded from IOPscience. Please scroll down to see the full text article.

2013 J. Phys. D: Appl. Phys. 46205303

(http://iopscience.iop.org/0022-3727/46/20/205303)

View the table of contents for this issue, or go to the journal homepage for more

Download details:

IP Address: 159.226.231.70

The article was downloaded on 07/06/2013 at 09:14

Please note that terms and conditions apply. 


\title{
Adhesive contact between a graphene sheet and a nano-scale corrugated surface
}

\author{
Hao Chen, Yin Yao and Shaohua Chen \\ LNM, Institute of Mechanics, Chinese Academy of Sciences, Beijing 100190, People's Republic of China \\ E-mail: chenshaohua72@hotmail.com
}

Received 24 January 2013, in final form 20 March 2013

Published 1 May 2013

Online at stacks.iop.org/JPhysD/46/205303

\begin{abstract}
Adhesive contacts between graphene sheets and corrugated surfaces are investigated. It is found that the final configuration between the graphene sheet and the substrate depends not only on the surface roughness of the substrate, but also on the length of graphene. A continuous transition, rather than a recent observation of 'snap-through' transition, is exhibited in our study. For a graphene sheet with a fixed length, it is easy to fully conform to the substrate of small roughness. Otherwise, the graphene sheet will remain flat on top of the corrugated substrate due to the unsatisfied bending energy or partially conform to the substrate due to the resistance of large interface friction. In order to reduce the effect of interface friction on the adhesive configuration, a new method, i.e. tilting the graphene sheet with a proper angle, is proposed. The tilting angle will significantly influence the final conformation of the adhesive interface. Some interesting types of behaviour are observed, such as rolling graphene, a double layer of graphene and fully adhesive contact, which is physically determined by the competition of thermal fluctuation and interfacial van der Waals interaction.
\end{abstract}

(Some figures may appear in colour only in the online journal)

\section{Introduction}

Graphene (GE) is a two-dimensional crystal consisting of hexagonally arranged, covalently bonded carbon atoms. Its exceptional properties, such as the extraordinary electrical [1], mechanical [2,3] and thermal [4] ones, give it great potential for applications, e.g. in nano-electronics [5], nanoelectromechanical systems [6], composite materials [7, 8] and surface modifications [9]. Due to the extremely small thickness and flexibility, GE is often used with other materials as a coating layer. Directly, GE can be an effective layer to protect the substrate from corrosion and oxidation, reduce the friction and enhance the wear behaviour, as a coating on other materials [9-14]. On the other hand, the combination of GE and other materials could form the basis of substrateregulated GE devices. No matter in what applications, adhesive interfaces between GE and other materials exist and are very important in tailoring GE's properties for device and materials. The morphology at the interface will in turn influence its electronic and mechanical properties [15-17]. Thus, many interesting works have been carried out in order to understand the morphology of the interface [18-23]. Recent experiments showed that the adhesion energy of pressurized monolayer/multilayer GE on top of a $\mathrm{SiO}_{2}$ substrate is ultra- strong $\left(0.45 \mathrm{~J} \mathrm{~m}^{-2}\right.$ for one layer and $0.3 \mathrm{~J} \mathrm{~m}^{-2}$ for multilayer $)$, which is many times larger than that reported for typical micromechanical structures and is of the order of a solidliquid adhesion energy [24], but the reason for the higher adhesion energy of a monolayer GE than a multilayer one was not adequately explained by these experimental studies. Some studies argued that the incomplete conformation of the latter to the rough substrate should be responsible for the lower adhesion energy [20-22], based on which theoretical models were developed to predict how the surface roughness affects the adhesion between GE membranes and substrates. Gao and Huang [21] found that the bending modulus, which increases drastically from monolayer to multilayered GE, plays an important role in the transition from conformal to nonconformal morphology of a GE membrane on a corrugated surface. Li and Zhang [20,23] suggested that there are only two equilibrium morphologies of GE: closely conforming to the substrate or remaining flat on the substrate, which was called the 'snap-through' phenomenon. Scharfenberg et al [19] revealed that GE undergoes a sharp 'snap-through' transition as a function of the layer thickness on a corrugated substrate. Wagner and Vella [22] demonstrated that substrate shapes should play a crucial role in determining the 'snapthrough' transition and proposed a substrate shape that could 


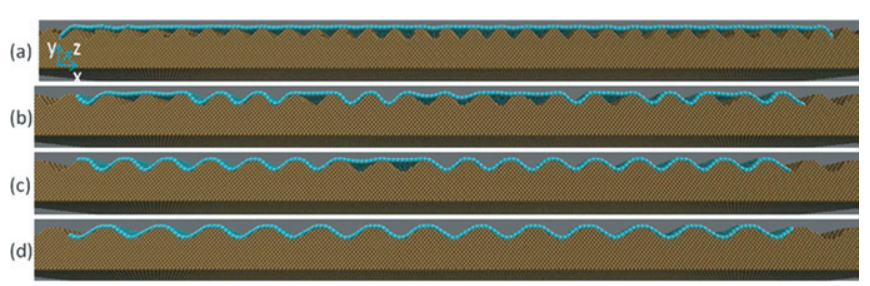

Figure 1. Side views of the stable configuration of a GE sheet of length $l_{x}=74.6 \mathrm{~nm}$ on top of a corrugated substrate with a fixed amplitude $a=0.5 \mathrm{~nm}$ but different wavelengths. The wavelengths are (a) $\lambda=2.5 \mathrm{~nm},(b) \lambda=3.5 \mathrm{~nm},(c) \lambda=4 \mathrm{~nm}$ and (d) $\lambda=4.5 \mathrm{~nm}$.

exhibit a continuous, rather than 'snap-through', transition. Neek-Amal and Peeters [18] used atomistic simulations to investigate morphological properties of monolayer GE with a fixed length deposited on a nanostructured substrate with different geometrical deformations.

Although there has been a great deal of discussion on GE morphology on a corrugated substrate, no final conclusions have been made and further studies are still required to consider some other influencing factors. In this paper, some important factors in a GE/substrate system are investigated with molecular dynamics (MD) simulations, mainly considering the effects of the length of GE and interfacial friction on the morphology of the adhesive interface. All the results in this paper should be helpful for a better understanding of physical interfaces between GE and substrates, and provide an alternative way for precise control of the adhesive interface morphology.

\section{Atomistic model}

In the MD simulation model, as shown in figure 1(a), a Cartesian coordinate $(x, y)$ is introduced and the $x$-axis is parallel to the zigzag direction. The out-of-plane coordinate is $z$. The surface roughness of the substrate is described by a sinusoidal function without loss of generality [25], which has often been adopted in theoretical and experimental studies on adhesion between GE and corrugated substrates:

$$
y=h_{0}+a \sin (k x)
$$

where $h_{0}$ is a constant, $a$ is the amplitude of the roughness, $\lambda$ is the wavelength and $k=2 \pi / \lambda$ is the wave number. Copper is chosen as a representative substrate with a thickness in the $y$ direction larger than $(2 a+3) \mathrm{nm}$ in our simulations and the width of the GE sheet in the $z$ direction is fixed as $11.2 \mathrm{~nm}$ in all the calculations. Atoms at the bottom of the substrate remain fixed. Periodic boundary conditions are applied in the $x$ and $z$ directions.

All the MD simulations in this paper are performed using the large-scale atomic molecular massively parallel simulator (LAMMPS) [26]. The adaptive intermolecular reactive empirical bond order (AIREBO) [27] potential is used to describe the interaction among carbon atoms, which gives a precise description of the bond-bond interaction, bond breaking and bond reforming of carbon atoms [28]. The cutoff parameter is taken as $2.0 \AA$ for REBO of the potential [29].
The embedded atom potential is used to describe the $\mathrm{Cu}-$ $\mathrm{Cu}$ atom interactions [30]. The classical Lennard-Jones (L-J) potential is adopted to describe interactions between carbon and copper atoms at the interface, which has been proved to be able to describe the interactions between two kinds of atoms precisely [31,32], i.e. $V(r)=4 \varepsilon\left(\sigma^{12} / r^{12}-\sigma^{6} / r^{6}\right)$. Here, $r$ is the distance between two atoms, $\varepsilon$ is a parameter determining the depth of the potential well and $\sigma$ is a length scale parameter that determines the position of the minimum potential. In the simulations, we take $\varepsilon=0.0168 \mathrm{eV}$ and $\sigma=$ $2.2 \AA$. Unless stated otherwise, all simulations are calculated in the NVT ensemble at temperature $10 \mathrm{~K}$. The equations of motion are solved using a velocity-Verlet algorithm with a time step of $1.0 \mathrm{fs}$. A Nose-Hoover thermostat is used to control the temperature of the system. In order to obtain a finally stable morphology, the simulation system is relaxed until the potential energy of the system does not change any more in the calculations.

\section{Results and discussion}

The initial morphology is monolayer GE lying horizontally on the top of the $\mathrm{Cu}$ substrate, as shown in figure 1(a). We take a fixed GE length $l_{x}=74.6 \mathrm{~nm}$, the amplitude $a$ as $0.5 \mathrm{~nm}$ and vary the wavelength $\lambda$ from 2 to $6 \mathrm{~nm}$. The simulation system is relaxed for sufficient time until a stable and minimum potential energy of the system is achieved. At this moment, GE will find an optimum configuration on the corrugated substrate as shown in figure 1 for cases with different wavelengths of the surface roughness. Four typical final morphologies of GE on a $\mathrm{Cu}$ substrate correspond to four kinds of wavelengths of the rough substrate: $\lambda=2.5 \mathrm{~nm}, \lambda=3.5 \mathrm{~nm}, \lambda=4 \mathrm{~nm}$ and $\lambda=5 \mathrm{~nm}$, respectively. GE does not deform or bend in figure $1(a)$ and remains flat on the substrate. As $\lambda$ increases to $3.5 \mathrm{~nm}$ in figure $1(b)$, GE begins to partially follow the substrate roughness. Further increasing the wavelength leads to an increasing length of GE conforming to the corrugated substrate, as shown in figure $1(c)$. When $\lambda$ is equal to $4.5 \mathrm{~nm}$, GE fully conforms to the corrugated substrate, as shown in figure $1(d)$. Comparing the morphologies in figures $1(a)-(d)$, one can see that the smoother the substrate, the more easily a full conformation of GE can be achieved, which agrees well with the numerical predictions in [18]. In contrast to the 'snap-through' phenomenon revealed in [19, 20,22], i.e. the material transitions between conforming to the substrate and lying flat on top of the substrate, a partially conformed state, as shown in figures $1(b)$ and $(c)$, can be found in our simulations. The classical theory of elasticity requires that since the thickness of a GE monolayer is essentially zero then the flexural rigidity should also be zero. However, atomistic scale simulations predict that the bond-angle effect on the interatomic interactions should result in a finite flexural rigidity defined by the interatomic potential used [33]. So from the energy conservation point of view, the final morphology should be a competition result among the bending and stretching energies of the GE sheet and the adhesion energy of the interface. If the adhesion energy of the interface is larger than the bending and stretching energies stored in the GE sheet, a 
Table 1. Binding energy of GE on corrugated substrates with a fixed amplitude $a=0.5 \mathrm{~nm}$ but different wavelengths $\lambda . E_{\text {bind } 1}$ is for GE with one layer and $E_{\text {bind2 }}$ is for GE with two layers.

\begin{tabular}{lll}
\hline$\lambda(\mathrm{nm})$ & $E_{\text {bind } 1}\left(\mathrm{eV} \mathrm{nm}^{-2}\right)$ & $E_{\text {bind2 }}\left(\mathrm{eV} \mathrm{nm}^{-2}\right)$ \\
\hline 2.5 & 0.8 & - \\
3.5 & 1.41 & 0.86 \\
4.0 & 1.72 & 1.24 \\
4.5 & 1.85 & 1.64 \\
5.0 & 1.86 & 1.93 \\
5.5 & 1.86 & - \\
6.0 & 1.87 & 1.98 \\
\hline
\end{tabular}

full conforming configuration will be achieved. Otherwise, a partial conformation, even a flat one, will be obtained. In particular, the bending energy stored in the GE sheet depends significantly on the surface roughness of the substrate. For the case of roughness with a fixed amplitude, the larger the wavelength of the roughness, the smaller the bending energy needed for GE to bend and conform to the rough surface. In our present simulation, for a fixed length $l=74.6 \mathrm{~nm}$ of GE and a fixed amplitude $a=0.5 \mathrm{~nm}$ of the $\mathrm{Cu}$ substrate roughness, the critical wavelength of the substrate roughness is $\lambda=4.5 \mathrm{~nm}$, which means the adhesion energy of the interface is nearly equal to the summation of the bending and stretching energies in the GE sheet in this case. Over the scale of $\lambda=4.5 \mathrm{~nm}$, it is more easier for monolayer GE to fully conform to the corrugated substrate because the bending energy and the stretching energy resisting the full conformation are smaller. However, if the wavelength is less than $4.5 \mathrm{~nm}$, monolayer GE may partially conform to or lie flat on the corrugated substrate.

The adhesion energies of the interface are given in table 1 for several cases, where the interface adhesion energy is shown to depend obviously on the final interface morphology and mainly on the adhesion area. When the interface morphology tends to be stable, the interface adhesion energy also converges to a constant. It is found that the total adhesion energy in each case increases with increasing relaxing time and finally tends to be a constant when the morphology is stable. Furthermore, the interface adhesion energy in the final interface morphology increases with an increasing $\lambda$ and also tends to be a constant if the wavelength is sufficiently large. This constant should be the value of adhesion energy in the case of a flat substrate. The adhesion energy of the case where the GE sheet lies flat on a rough substrate is the smallest, while that in the fully conforming configuration achieves the maximum, as shown in table 1.

The adhesion morphology of a two-layer GE on a corrugated substrate is also investigated. Figure 2 shows the final morphologies of a two-layer GE on a substrate, where the two-layer GE sheet has a fixed length $l=74.6 \mathrm{~nm}$ and the determined amplitude of the substrate roughness is $a=0.5 \mathrm{~nm}$ but with different wavelengths. Similar to the monolayer case, the final stable morphologies are different due to the different wavelengths of substrate. The two-layer GE will lie flat on top of the corrugated substrate when the wavelength of the substrate is $3.5 \mathrm{~nm}$, then experience a partial conformation to the substrate when the wavelength increases to 4.0 and

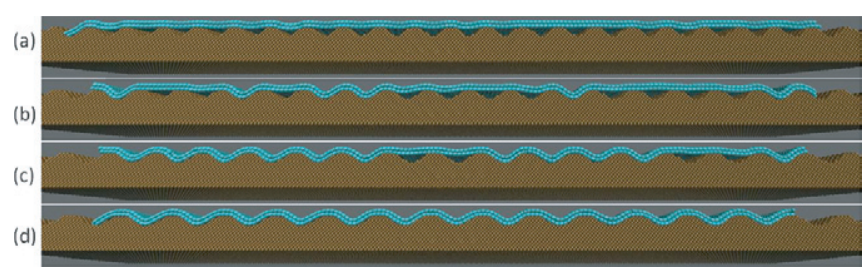

Figure 2. Side views of the stable configuration of a two-layer GE sheet of length $l_{x}=74.6 \mathrm{~nm}$ on top of a corrugated substrate with a fixed amplitude $a=0.5 \mathrm{~nm}$ but different wavelengths. The wavelengths are $(a) \lambda=3.5 \mathrm{~nm},(b) \lambda=4.0 \mathrm{~nm},(c) \lambda=4.5 \mathrm{~nm}$ and $(d) \lambda=5 \mathrm{~nm}$

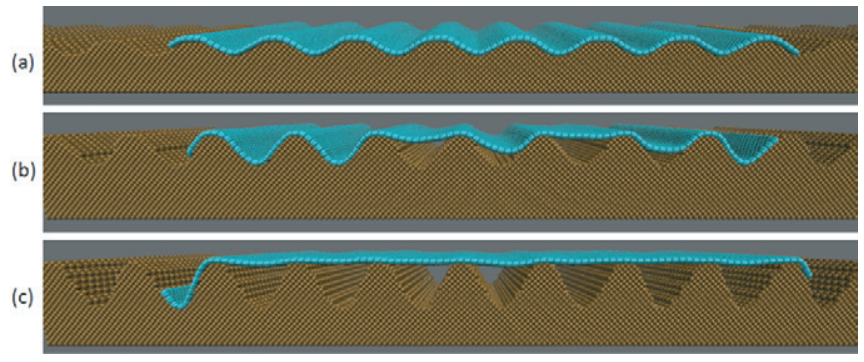

Figure 3. Side views of the stable configuration of a monolayer GE sheet of length $l_{x}=42.7 \mathrm{~nm}$ on top of a corrugated substrate with a fixed wavelength $\lambda=5.0 \mathrm{~nm}$ but different amplitudes: (a) $a=0.5 \mathrm{~nm}$, (b) $a=1.0 \mathrm{~nm}$ and (c) $a=1.5 \mathrm{~nm}$.

$4.5 \mathrm{~nm}$. Full conforming configuration of the interface is achieved when the wavelength of the substrate is $5.0 \mathrm{~nm}$. It is found that the critical wavelength of the substrate roughness is about $\lambda=5.0 \mathrm{~nm}$, over which a two-layer GE sheet with a fixed length $l=74.6 \mathrm{~nm}$ can fully conform to the corrugated substrate and below which GE may partially conform to or lie flat on the corrugated substrate.

Comparing the monolayer case with the two-layer case, even a multilayer one, we find that the critical wavelength of the substrate increases with the number of layers of GE if the length of GE and the amplitude of the substrate are fixed in differentlayer cases. Since the bending stiffness of a GE sheet increases with the number of its layer [21], more bending energy will be needed for the GE sheet with an increasing number of layers to achieve full conformation to a corrugated substrate, which is consistent with the experimental results [24].

In order to investigate the effect of the amplitude of substrate roughness on the final interface configuration, we consider another case with the same length $l_{x}=42.7 \mathrm{~nm}$ and the same wavelength $\lambda=5.0 \mathrm{~nm}$ but different amplitudes, as shown in figure 3. It is found that, with an increasing amplitude, the stable morphology would change from a full conformation in figure $3(a)$ to a partial one in figure $3(b)$ and finally remain flat on the substrate, as shown in figure 3(c). The larger the amplitude of the substrate roughness, the larger the bending energy required for the GE sheet to conform to the corrugated surface when the wavelength of the substrate roughness remains unchanged.

If the surface roughness of the substrate is fixed, i.e. unchanged amplitude and wavelength, does the length of the GE sheet influence the final adhesive interface morphology? Here, a series of simulations are carried out, in which the GE sheet has different lengths and the amplitude of substrate 


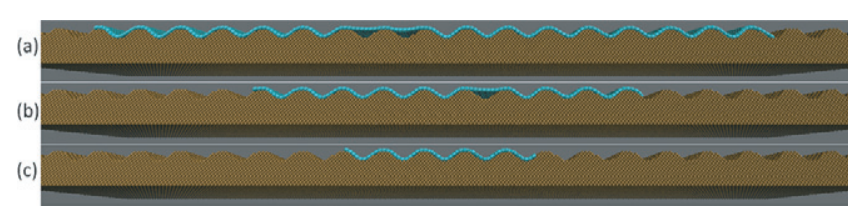

Figure 4. Side views of the stable configuration of a GE sheet of different lengths on top of a corrugated substrate with a fixed amplitude $a=0.5 \mathrm{~nm}$ and a determined wavelength $\lambda=4 \mathrm{~nm}$. The lengths of GE are (a) $l_{x}=74.6 \mathrm{~nm},(b) l_{x}=42.7 \mathrm{~nm}$ and (c) $l_{x}=21.4 \mathrm{~nm}$.

(a)

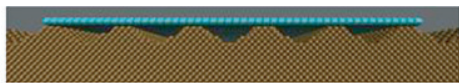

(b)

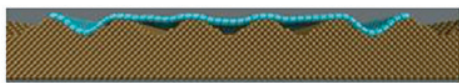

(c)

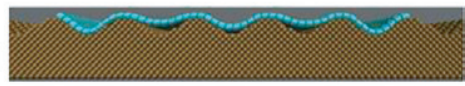

(d)

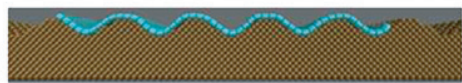

Figure 5. Snapshots of the conformation process, where $t$ is the relaxation time of the system. The length of GE is $21.4 \mathrm{~nm}$. The wavelength of the substrate surface is $4 \mathrm{~nm}$ and the amplitude is $a=0.5 \mathrm{~nm}$. The relaxation times are $(a) t=0 \mathrm{ps},(b) t=7.2 \mathrm{ps}$, (c) $t=9.6 \mathrm{ps}$ and $(d) t=18 \mathrm{ps}$.

roughness is fixed as $a=0.5 \mathrm{~nm}$ with the wavelength $\lambda=$ $4 \mathrm{~nm}$. The simulation results are given in figure 4 for three cases with lengths of GE equal to $74.6 \mathrm{~nm}, 42.7 \mathrm{~nm}$ and $21.4 \mathrm{~nm}$, respectively. It is found that the shorter the length of GE, the more easily a full conformation can result. Simulations for a two-layer or a multilayer GE case with different lengths are also conducted. With the same wavelength of the substrate, the length of GE to obtain a fully conforming interface configuration will decrease with an increasing layer of GE. In a word, the final interface morphology depends significantly on the length and the number of layers of GE. The more layers the GE sheet has, the shorter the length of GE to achieve full conformation to the corrugated substrate. The effect of layer of GE on the final morphology is easy to understand, being due to the bending stiffness of the GE sheet. However, how does the length of GE influence the final morphology?

Figure 5 shows the snapshots of a monolayer GE sheet of length $21.4 \mathrm{~nm}$ on a corrugated substrate. At the initial time, the monolayer GE lies flat on the corrugated substrate. At the relaxed time $t=7.2 \mathrm{ps}$, the monolayer GE starts to conform to the substrate from two ends. Afterwards, GE slides on the substrate from two ends to the middle and finally, full conformation morphology is achieved at the relaxed time $t=18 \mathrm{ps}$. Snapshots of a monolayer GE sheet with a relatively larger length on a corrugated substrate are also checked. Similar to the case with a short GE, conformation to the substrate also starts from the two ends of GE and then GE slides on the substrate to achieve a larger conformation length to the substrate. When the length of the conformation region is large enough, GE cannot slide any longer and partial conformation morphology forms. All the phenomena denote that the interfacial friction should be an important factor, which leads to the apparent effect of GE length on

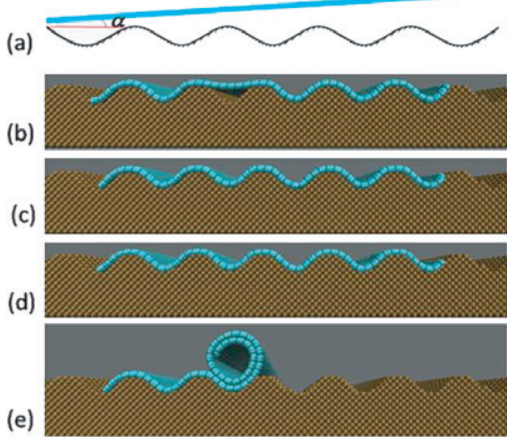

Figure 6. Final morphologies of GE of length $l_{x}=21.4 \mathrm{~nm}$ on a corrugated substrate with $a=0.5 \mathrm{~nm}$ and $\lambda=3.5 \mathrm{~nm}$.

(a) Schematic of a GE sheet with a tilted angle $\alpha$ on top of a corrugated substrate. The tilted angles of GE are $(b) \alpha=5^{\circ},(c)$ $\alpha=10^{\circ},(d) \alpha=20^{\circ}$ and $(e) \alpha=30^{\circ}$.

the final morphology and thus a continuous transition in MD simulations. In the previous theoretical work [20-22], only the bending energy of GE and the interfacial adhesion energy between GE and the substrate are considered. The competition between the van der Waals interaction and the bending energy determines the equilibrium morphology [20-22]. However, in real fabrications, the friction force would have an influence on the final configuration, where the two ends of GE conform to the substrate first, and then GE will slide on the corrugated surface. The work of friction force will consume a part of the energy, which may lead to a partially conformed interface configuration.

Simulations with different temperatures are also considered, where we find an insignificant effect of temperature on the final results. Details are omitted here.

The effect of interfacial friction in the case of a relatively long GE sheet is so significant that it is not easy to obtain a full conformation interface configuration. A method of tilting the GE sheet at an angle $\alpha$ with the horizontal direction is considered, as shown in figure 6(a), which has been found to be feasible in transferring techniques [34]. However, how large should the tilting angle be and what are the other factors influencing the final configurations in this method? Details are discussed in our MD simulations. In figure 6(a), one end of the tilted GE tends to conform to the corrugated substrate, while the other end, out of the range of van der Waals interaction, exhibits free-standing behaviour. Corrugation in the freestanding GE end forms spontaneously owing to the thermal fluctuation, which is therefore random [35]. Figures 6(b)(e) illustrate the final morphologies of $\mathrm{GE}$ on a corrugated substrate, where GE has a length of $l_{x}=21.4 \mathrm{~nm}$ and an initially tilted angle $\alpha$, on an identical substrate with roughness parameters $\lambda=3.5 \mathrm{~nm}, a=0.5 \mathrm{~nm}$. When the tilted angle is small, e.g. $\alpha=5^{\circ}$, the free-standing part falls rapidly and the two ends of the GE sheet conform to the substrate easily with a non-conformal part. As the tilted angle increases, GE could fully conform to the corrugated substrate very well, e.g. $\alpha=10^{\circ}$ and $20^{\circ}$, as shown in figures $6(c)$ and $(d)$, respectively. However, the tilted angle increases further, the free-standing part of GE will roll spontaneously and form a stable cylindrical configuration on the substrate. A comparison of different 


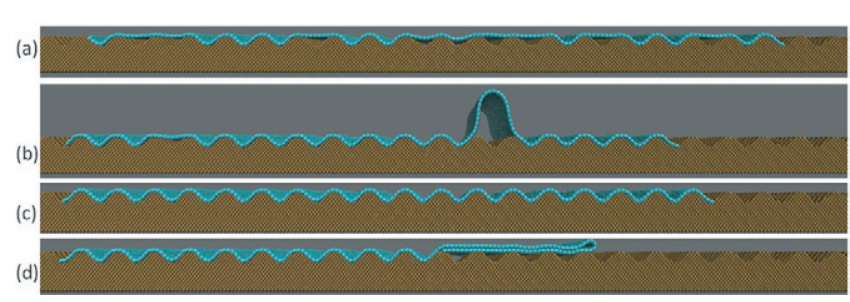

Figure 7. Final morphologies of GE of length $l_{x}=74.6 \mathrm{~nm}$ on a corrugated substrate with $a=0.5 \mathrm{~nm}$ and $\lambda=3.5 \mathrm{~nm}$. The tilted angles of GE are (a) $\alpha=0^{\circ}$, (b) $\alpha=3^{\circ},(c) \alpha=5^{\circ}$ and (d) $\alpha=10^{\circ}$.

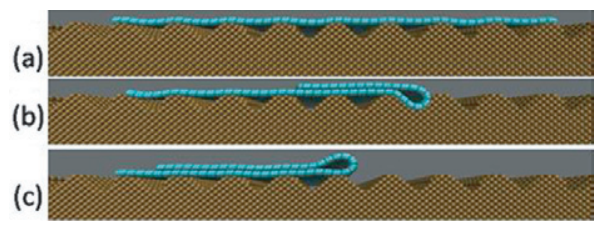

Figure 8. Final morphologies of GE of length $l_{x}=21.4 \mathrm{~nm}$ on a corrugated substrate with $a=0.5 \mathrm{~nm}$ and $\lambda=2.5 \mathrm{~nm}$. The tilted angles of GE are (a) $\alpha=5^{\circ},(b) \alpha=15^{\circ}$ and $(c) \alpha=30^{\circ}$.

cases in figure 6 demonstrates that the competition of the separation induced by the thermal fluctuation and attraction resulting from the van der Waals interaction will determine the final morphology of the interface. If the tilted angle is very small, interface friction cannot be avoided. On the other hand, if the tilted angle is very large, the interaction of van der Waals force between the free-standing part of GE and the corrugated substrate is very weak and the thermal fluctuation will dominate, which may induce a self-assembly of the GE sheet, e.g. the cylindrical form in figure $6(d)$.

Now consider the case where the substrate roughness remains the same as in figure 6 and only the length of the GE sheet is changed, as shown in figure 7 , where GE has a length of $74.6 \mathrm{~nm}$. If the tilted angle is zero, i.e. $\alpha=0^{\circ}$, GE cannot fully conform to the corrugated substrate, as shown in figure 7(a). As the tilted angle increases to $\alpha=3^{\circ}$, the length of the non-conformal part of GE decreases, as shown in figure $7(b)$. When the tilted angle is $5^{\circ}$, it is interesting to find that GE can fully conform to the corrugated substrate very well, as shown in figure $7(c)$. However, figure $7(d)$ exhibits that the free-standing part of GE will self-fold on the substrate when the tilted angle is $10^{\circ}$ or larger. The results in figure 7 demonstrate that the tilting method could be used to achieve a full conformation configuration if only the tilted angle is chosen properly, which should be related to the length of GE. If the length of GE is large, the proper tilted angle may be small. Otherwise, the free-standing part will be dominated by thermal fluctuation first, even if the left-hand side part, as shown in figure $7(d)$, will conform to the corrugated substrate little by little from the left to the right due to the van der Waals interaction. However, if the tilted angle is very small, the final configurations as shown in figures 7(a) and $(b)$ will be found, which is due to the coupling and competing effects of van der Waals interaction and the thermal fluctuation.

Now consider the case where the length of GE and the amplitude of the substrate roughness remain the same as those in figure 5 and only the wavelength of the substrate roughness is changed, as shown in figure 8 , where the wavelength is $\lambda=2.5 \mathrm{~nm}$. We find that, although the tilted angle is changed arbitrarily, two kinds of morphologies are observed: GE remaining flat on the substrate or folding on the substrate to form a double layer. It is not surprising since GE remains flat on such a substrate even if the tilted angle vanishes and the interface friction does not influence the final configuration. Only in the case with a relatively long GE sheet, in which partial conformation can be formed and the interface friction will resist the sliding of GE on the corrugated substrate, will the method of tilting the GE sheet by a proper angle on the corrugated substrate work in order to obtain full conformation morphology.

\section{Conclusion}

In conclusion, atomistic molecular simulations are carried out in order to study the stable configuration of GE in adhesive contact with a corrugated substrate. It is found that the final optimum configuration depends not only on the surface roughness of the substrate but also on the length of GE. A continuous transition of the morphology of GE on a corrugated substrate is found, i.e. GE remaining flat on the substrate, partial conformation to the substrate and full conformation configuration, which is different from the recent observations of a sharp 'snap-through' transition [20,22,23], i.e. from flat to conforming states. Especially, in the case where a short GE sheet could fully conform to the substrate, but partial conformation morphology will form due to the relatively larger length of GE, a method of tilting GE is proposed to reduce the effect of interface friction. It is found that a region of tilting angle exists, in which the long GE sheet could realize full conformation to the corrugated substrate. The final configuration depends on the competition of the separation induced by thermal fluctuation and the attraction resulting from van der Waals interaction. If the tilted angle is large, thermal fluctuation dominates the free-standing end of GE, which will lead to a rolling structure or a double layer of GE on a corrugated substrate.

\section{Acknowledgments}

The work reported here is supported by the NSFC through Grants \#10972220, \#11125211, \#11021262 and the Nanoproject (2012CB937500).

\section{References}

[1] Novoselov K S, Geim A K, Morozov S V, Jiang D, Katsnelson M I, Grigorieva I V, Dubonos S V and Firsov A A 2005 Nature 438 197-200

[2] Bunch J S, van der Zande A M, Verbridge S S, Frank I W, Tanenbaum D M, Parpia J M, Craighead H G and McEuen P L 2007 Science 315 490-3

[3] Lee C, Wei X D, Kysar J W and Hone J 2008 Science $321385-8$

[4] Balandin A A, Ghosh S, Bao W Z, Calizo I, Teweldebrhan D, Miao F and Lau C N 2008 Nano Lett. 8 902-7

[5] Bae S et al 2010 Nature Nanotechnol. 5 574-8 
[6] Chen C Y, Rosenblatt S, Bolotin K I, Kalb W, Kim P, Kymissis I, Stormer H L, Heinz T F and Hone J 2009 Nature Nanotechnol. 4 861-7

[7] Zhang H, Lv X, Li Y, Wang Y and Li J 2009 ACS Nano $4380-6$

[8] Chou S L, Wang J Z, Choucair M, Liu H K, Stride J A and Dou S X 2010 Electrochem. Commun. 12 303-6

[9] Chen S S et al 2011 ACS Nano 5 1321-7

[10] Hao Q Z, Wang B, Bossard J A, Kiraly B, Zeng Y, Chiang I K, Jensen L, Werner D H and Huang T J 2012 J. Phys. Chem. C $1167249-54$

[11] Lee J S, Ahn H J, Yoon J C and Jang J H 2012 Phys. Chem. Chem. Phys. 14 7938-43

[12] Mi Y J, Wang Z F, Liu X H, Yang S R, Wang H G, Ou J F, Li Z P and Wang J Q 2012 J. Mater. Chem. 22 8036-42

[13] Prasai D, Tuberquia J C, Harl R R, Jennings G K and Bolotin K I 2012 ACS Nano 6 1102-8

[14] Tuberquia J C, Harl R R, Jennings G K, Rogers B R and Bolotin K I 2012 ACS Nano 64540

[15] Ferrari A C et al 2006 Phys. Rev. Lett. 97187401

[16] Ishigami M, Chen J H, Cullen W G, Fuhrer M S and Williams E D 2007 Nano Lett. 7 1643-8

[17] Stolyarova E, Rim K T, Ryu S M, Maultzsch J, Kim P, Brus L E, Heinz T F, Hybertsen M S and Flynn G W 2007 Proc. Natl Acad. Sci. USA 104 9209-12

[18] Neek-Amal M and Peeters F M 2012 Phys. Rev. B 85195445
[19] Scharfenberg S, Mansukhani N, Chialvo C, Weaver R L and Mason N 2012 Appl. Phys. Lett. 100021910

[20] Li T and Zhang Z 2010 J. Phys. D: Appl. Phys. 43075303

[21] Gao W and Huang R 2011 J. Phys. D: Appl. Phys. 44452001

[22] Wagner T J W and Vella D 2012 Appl. Phys. Lett. 100233111

[23] Zhang Z and Li T 2011 J. Appl. Phys. 110083526

[24] Koenig S P, Boddeti N G, Dunn M L and Bunch J S 2011 Nature Nanotechnol. 6 543-6

[25] Chung S, Lee J H, Moon M W, Han J and Kamm R D 2008 Adv. Mater. 20 3011-6

[26] Plimpton S 1995 J. Comput. Phys. 117 1-19

[27] Stuart S J, Tutein A B and Harrison J A 2000 J. Chem. Phys. 112 6472-86

[28] Zhao H and Aluru N R 2010 J. Appl. Phys. 108064321

[29] Belytschko T, Xiao S P, Schatz G C and Ruoff R S 2002 Phys. Rev. B 65235430

[30] Foiles S M, Baskes M I and Daw M S 1986 Phys. Rev. B 33 7983-91

[31] Guo Y F, Kong Y, Guo W L and Gao H J 2004 J. Comput. Theor. Nanosci. $193-8$

[32] Guo Y F and Guo W L 2006 Nanotechnology 17 4726-30

[33] Huang Y, Wu J and Hwang K C 2006 Phys. Rev. B 74245413

[34] Calado V E, Schneider G F, Theulings A M M G, Dekker C and Vandersypen L M K 2012 Appl. Phys. Lett. 101103116

[35] Meyer J C, Geim A K, Katsnelson M I, Novoselov K S, Booth T J and Roth S 2007 Nature 446 60-3 